\title{
Down-regulation of lipoprotein lipase increases ABCA1- mediated cholesterol efflux in THP-1 macrophages
}

\author{
Ryoko L. Kawashima and Jheem D. Medh \\ Department of Chemistry and Biochemistry, California State University Northridge Northridge CA \\ 91330-8262
}

\section{Abstract}

The ATP-binding cassette transporter A1 (ABCA1) mediates the efflux of excess cholesterol from foam cells to lipid-poor apolipoprotein A-I, in a process called reverse cholesterol transport. Lipoprotein lipase (LPL) is a lipolytic enzyme expressed by macrophages within atherosclerotic lesions. Lentivirus-mediated RNA interference was used to genetically knock-down (KD) the expression of LPL in THP-1 macrophages. Silencing of the LPL gene was confirmed by end-point PCR, real time PCR, and protein analysis. Suppression of LPL expression correlated with a 1.6fold up-regulation of ABCA1 mRNA levels, and resulted in a 4.5-fold increase in ABCA1dependent cholesterol efflux. Replenishing LPL by addition of purified bovine LPL to the cell culture media resulted in down-regulation of ABCA1-mediated cholesterol efflux in both wildtype and LPL knockdown cells. These finding suggests an inverse correlation between macrophage LPL levels and ABCA1 cholesterol transport activity.

\section{Keywords}

ABCA1 transporter; lipoprotein lipase; cholesterol efflux; reverse cholesterol transport; THP-1 macrophages; shRNA

\section{INTRODUCTION}

\begin{abstract}
Atherosclerosis is initiated when circulating monocytes adhere to the vascular wall, enter the intimal space and ingest copious amounts of lipids to differentiate into large macrophagederived foam cells [1]. High density lipoproteins (HDL) protect against atherosclerosis by facilitating the removal of excess free cholesterol from vascular cells. Four different mechanisms have been described for the transport of cholesterol from cells [2]: (i) aqueous diffusion down a concentration gradient, (ii) bidirectional flux mediated by scavenger receptor class-B type I (SR-BI), (iii) ATP-binding cassette transporter A1 (ABCA1)mediated unidirectional efflux of cholesterol and phospholipids to lipid-poor apolipoprotein
\end{abstract}

\footnotetext{
(C) 2014 Elsevier Inc. All rights reserved.

*Corresponding author: Jheem D. Medh, jheem.medh@ csun.edu, Telephone: 818-677-7737.

Publisher's Disclaimer: This is a PDF file of an unedited manuscript that has been accepted for publication. As a service to our customers we are providing this early version of the manuscript. The manuscript will undergo copyediting, typesetting, and review of the resulting proof before it is published in its final citable form. Please note that during the production process errors may be discovered which could affect the content, and all legal disclaimers that apply to the journal pertain.
} 
acceptors such as apolipoprotein A-I (apoA-I) and apolipoprotein E (apoE), and (iv) ABCG1-mediated unidirectional efflux to nascent HDL particles. Both ABCA1 and ABCG1 are highly expressed in macrophages whereas $\mathrm{SR}-\mathrm{BI}$ is mainly expressed in steroidogenic tissues and liver. Hence, the ABCA1 and ABCG1 pathways are the dominant cholesterol efflux mechanism in macrophages.

ABCA1 is a member of a large family of transporters that have nucleotide binding domains and hydrolyze ATP (are ATPases) for energy to transport various substrates including lipids, ions and metabolites [3]. ABCA1-mediated cholesterol efflux has an absolute requirement for an apolipoprotein acceptor [4]. ABCA1 levels are tightly regulated in order to maintain lipid homeostasis. Cholesterol-loading and cAMP analogues are major positive regulators of ABCA1 expression [5]. The transcription of ABCA1 gene is also highly up-regulated by the activation of nuclear receptors Liver X Receptor (LXR), retinoid X receptor (RXR), and peroxisome proliferators activated receptor (PPAR) [6].

Macrophages abundantly express another protein, lipoprotein lipase (LPL), a secreted lipolytic enzyme that facilitates the hydrolysis of triglycerides (TG) in chylomicrons to release fatty acids for energy or storage [7,8]. Depletion of TG converts chylomicrons into cholesterol-rich remnant particles which are ligands for macrophage lipoprotein receptors and are instrumental in macrophage cholesterol accumulation. This mechanism may partially explain the contribution of macrophage LPL to atherogenesis [7]. Several studies have demonstrated that LPL is abundantly expressed in macrophage-derived foam cells lodged within atherosclerotic lesions [7]. Animal models with macrophage specific overexpression of human LPL point to a role for LPL in cholesterol accumulation and lesion growth [9-12]. While the positive correlation between macrophage LPL and atherosclerosis is undisputed, the specific cellular events or mechanisms by which LPL precipitates fatty lesions are not well understood. Since ABCA1 is a key player in cellular cholesterol homeostasis in macrophages, we hypothesized that macrophage LPL may regulate ABCA1 expression and function. Short interfering RNA (siRNA) technology was used to silence the LPL gene in THP-1 cells, a human acute monocytic leukemia cell line. Our data confirm that LPL levels inversely correlate with ABCA1 expression and cholesterol efflux in THP-1 macrophages.

\section{METHODS}

\section{Cell Culture and Differentiation}

THP-1 monocytes were obtained from ATCC and maintained in growth media (RPMI 1640 supplemented with10\% FBS (Atlanta Biologicals), 50units $/ \mathrm{mL}$ penicillin, $50 \mu \mathrm{g} / \mathrm{mL}$ streptomycin, $10 \mathrm{mM}$ HEPES, $\mathrm{pH} 7.4,2 \mathrm{mM}$ glutamine, $1 \mathrm{mM}$ sodium pyruvate, and $50 \mu \mathrm{M} \beta$ mercaptoethanol) at $37{ }^{\circ} \mathrm{C}$ and $5 \% \mathrm{CO}_{2}$. Monocytes were differentiated to macrophages in differentiation media (growth media without FBS, supplemented with $1 \mathrm{mg} / \mathrm{mL}$ BSA and 200nM phorbol-12-myristate-13-acetate (PMA)) within 48-72 hr as evidenced by their adherence to the culture plate. 


\section{Silencing the LPL Gene}

Wild-type (WT) THP-1 monocytes were seeded into two T25 $\mathrm{cm}^{2}$ tissue culture flasks in growth medium at a density of $1 \times 10^{5}$ cells $/ \mathrm{mL}$. The next day, the cells were resuspended in $5 \mathrm{~mL}$ of growth media supplemented with $5 \mu \mathrm{g} / \mathrm{mL}$ polybrene. LPL shRNA lentivirus (Santa Cruz Biotechnology, $0.5 \times 10^{6}$ infectious units of virus in $100 \mu \mathrm{L}$ ) was added, cells were chilled for 15 minutes, and transferred to $37^{\circ} \mathrm{C}$. The control flask was handled identically with the omission of Lentivirus. After 48 hours, the viral load was removed by centrifugation, cells were washed with PBS, and cultured in growth media. Cells transfected successfully (designated LPL-KD THP-1 cells) were selected by treatment with $10 \mu \mathrm{g} / \mathrm{mL}$ puromycin until all cells in the control flask were confirmed dead.

\section{RNA Isolation and RT-PCR}

RNA was isolated using TRI reagent (Sigma) and Direct-zol ${ }^{\mathrm{TM}}$ RNA miniprep kit (Zymo Research) according to the manufacturers' protocols. RNA was quantified by spectrophotometry at $260 \mathrm{~nm}$ and $4 \mu \mathrm{g}$ of RNA was used to synthesize cDNA by reverse transcription using Moloney Murine Leukemia Virus Reverse Transcriptase (M-MLV RT), dNTPs, and oligodT primers (Promega).

End-point PCR was performed using cDNA and primer pairs shown (Table 1). The PCR amplicons were resolved by $2 \%$ agarose gel and the DNA bands were quantified by ImageJ (NIH) analysis. The cDNA was also subjected to real time quantitative PCR using a Smart cycler (Cepheid Inc), RealMasterMix (5PRIME), and primer pairs shown (Table 1). A melting temperature $\left(\mathrm{T}_{\mathrm{m}}\right)$ of $85^{\circ} \mathrm{C}$ or higher was obtained, confirming primer-specific amplification. $\beta$-actin was used as the house-keeping gene control for both conventional and quantitative PCR. The threshold cycle $\left(\mathrm{C}_{\mathrm{T}}\right)$ values were used to calculate fold change in transcript levels using the $2^{-\Delta \Delta C_{T}}$ method [13] as follows: Fold change $=$ $2-\left(\mathrm{C}_{\mathrm{T}} \text { target }-\mathrm{C}_{\mathrm{T}} \beta \text {-actin }\right)_{\text {siRNA }}-\left(\mathrm{C}_{\mathrm{T}} \text { target }-\mathrm{C}_{\mathrm{T}} \beta \text {-actin }\right)_{\text {control }}$

\section{Analysis of de novo LPL Protein Synthesis}

The level of LPL protein translation was compared in WT and LPL-KD THP-1 macrophages by pulse-chase labeling. This protocol tags only de novo synthesized metabolites during biosynthesis. WT and LPL-KD THP-1 monocytes were plated at $2.5 \times 10^{6}$ cells per well on a 12-well tissue culture plate and differentiated as above. The cells were depleted of methionine by incubation in methionine-free minimum essential medium (MEM) for 30 minutes, and then incubated with $200 \mu \mathrm{Ci} / \mathrm{mL}$ of ${ }^{35} \mathrm{~S}$-labeled methionine (radioactive label) in MEM for 4 hours. Any incompletely synthesized proteins were chased to completion using $100 \mu \mathrm{M}$ cold methionine (Sigma) supplemented with $100 \mathrm{units} / \mathrm{mL}$ heparin for 30 minutes. Heparin was added to allow the dissociation of LPL from the cell surface proteoglycans. The medium was collected and cleared of cellular debris while the cellular monolayer was solubilized using $150 \mu \mathrm{L} /$ well of lysis buffer $(0.1 \%$ Triton-X-100 in $50 \mathrm{mM}$ Tris. $\mathrm{HCl}, \mathrm{pH} 8.0$ ). Both media and lysates were adjusted to $10 \%$ glycerol and $0.05 \%$ Triton X-100.

A chicken anti-LPL $\operatorname{IgY}(2 \mu \mathrm{g} / \mathrm{mL})$ immobilized on goat anti-chicken $\operatorname{Ig} Y$-agarose was used to immunoprecipitate LPL from $1 \mathrm{~mL}$ media or $100 \mu \mathrm{L}$ of lysates, after ascertaining equal 
radioactivity in WT and LPL-KD samples. Equal volumes of immunoprecipitated samples were resolved by $10 \%$ SDS-PAGE. The gel was then dried between cellophane sheets and LPL protein bands were visualized by autoradiography.

\section{Cholesterol Efflux Assay}

Wild-type or LPL knock down (LPL-KD) THP- 1 cells were plated at $2.5 \times 10^{6}$ cells $/ \mathrm{mL}$ per well. They were differentiated as above for $48 \mathrm{hr}$. The differentiated macrophage monolayer was rinsed with PBS, and incubated with ${ }^{3} \mathrm{H}$-cholesterol labeled oxidized LDL (final $0.25 \mu \mathrm{Ci} / \mathrm{mL} /$ well and $50 \mu \mathrm{g}$ oxLDL $/ \mathrm{mL} /$ well) for $48 \mathrm{hr}$. The labeled oxidized LDL was prepared by drying $3 \mu \mathrm{Ci}$ of $\left[1,2-{ }^{3} \mathrm{H}(\mathrm{N})\right]$-cholesterol in toluene (Perkin Elmer) under nitrogen gas and re-suspending it in 500 $\mu \mathrm{L}$ of efflux media (growth media without FBS, supplemented with $1 \mathrm{mg} / \mathrm{mL}$ BSA) by sonication in a $23^{\circ} \mathrm{C}$ water bath for 8 minutes. To this, $600 \mu \mathrm{g}$ of ox-LDL was added and incubated on ice for 20 minutes. (Oxidized LDL was obtained by prolonged exposure of LDL isolated from human plasma by sequential density gradient centrifugations [14] to the atmosphere). This solution was transferred to $37^{\circ} \mathrm{C}$ for 20 minutes, returned to RT, before mixing it with efflux media for a final volume of $12 \mathrm{~mL}$. Each well of differentiated macrophages received $1 \mathrm{~mL}$ of this ${ }^{3} \mathrm{H}$-cholesterol labeled oxidized LDL. After two days the cell monolayer was rinsed with PBS $+1 \mathrm{mg} / \mathrm{mL}$ BSA. Next, cellular cholesterol pools were equilibrated by incubation for 48 hours in efflux media supplemented with unlabeled $20 \mu \mathrm{g} / \mathrm{mL}$ cholesterol. After washing with PBS $+1 \mathrm{mg} / \mathrm{mL}$ BSA, cholesterol efflux was initiated by incubation in efflux media in the presence or absence of $32.7 \mu \mathrm{g} / \mathrm{mL}$ apolipoprotein-AI. After 12-14 hours, the media was collected and cellular debris was removed by centrifugation. The macrophage monolayer was washed twice with PBS/BSA, the cells were chilled and cellular lipids were extracted with 3:2 hexanes: isopropanol (v/v). Radioactivity in the efflux media and the cellular lipid extracts was measured by scintillation counting. The amount of cholesterol effluxed from the cell was calculated using the formula: \% Cholesterol Efflux $=[($ radioactivity in the medium $) /$ $\{$ (radioactivity in the medium $)+($ radioactivity in the lipid extracts $)\}] \times 100$. ABCA1mediated cholesterol efflux was estimated as the difference in cholesterol efflux in the presence and absence of apoAI acceptor.

\section{RESULTS}

\section{Creating LPL-Knock-Down THP-1 Cells}

In order to investigate the role of macrophage LPL in regulating ABCA1-mediated cholesterol efflux, we first genetically silenced the LPL gene in THP-1 cells. Lentivirus particles were used to deliver LPL-specific short hairpin RNA encoding plasmids into THP-1 monocytes. Stably transduced cells were selected by puromycin resistance. There was no difference in cell morphology between wild type (WT) and LPL-knock-down (LPLKD) cells. Silencing of the LPL gene was confirmed by end-point PCR, real time PCR, and Western blot analysis. As shown in Figure 1A, end-point RT-PCR failed to detect a LPLspecific amplicon in shRNA transduced cells indicating a complete silencing of the LPL gene. There was no difference in the intensity of a $\beta$-actin amplicon confirming that shRNA specifically targeted the LPL gene. $\beta$-actin also served as a control for RNA quantification and equal loading of the agarose gel. The down-regulation of LPL transcript was also 
confirmed by real-time quantitative PCR as shown in Table 2 . While the $\mathrm{C}_{\mathrm{T}}$ values for $\beta$ actin were similar in WT and LPL-KD cells, the $\mathrm{C}_{\mathrm{T}}$ value for LPL was significantly higher in LPLKD cells. Using the $2^{-\Delta \Delta C}$ T method, this was calculated to be only $0.3 \%$ of the transcript present in WT cells. Identical results were obtained after repeated experiments.

Next, the level of LPL protein was compared in wild-type and LPL-KD cells by metabolic labeling experiments. Since LPL is a secreted protein with a relatively long half-life, it accumulates in the cell culture media. Thus, to compare the level of LPL protein translation, metabolic labeling experiments were preferred to Western blotting. Radiolabeled LPL was immuno-precipitated from lysates and culture media of WT and LPL-KD cells. A band corresponding to LPL was detected in WT samples but not in LPL-KD samples (Figure 1B), indicating the absence of de novo LPL protein translation, consistent with the mRNA data in Figure 1A. Due to the short span of the experiment, all of the labeled LPL may not have traversed to the extracellular media. Hence, the intensity of the LPL band in cell lysates is stronger than that in the culture media. The slightly larger size of secreted LPL reflects its post-translational glycosylation prior to secretion.

\section{LPL Silencing Induces ABCA1 mRNA Expression in THP-1 Macrophages}

In previous studies, transgenic mice with macrophage-specific over-expression of human LPL showed a marked increase in atherosclerotic lesion size. This could be a result of increased cholesterol uptake, reduced cholesterol efflux, or both. This study was designed to investigate any correlation between LPL expression and cholesterol efflux. Thus, the expression of cholesterol transporters was compared in WT and LPL-KD cells by end-point RT-PCR (Figure 2A). Down-regulation of LPL correlated with a significant increase in ABCA1 expression ( $161 \%$ of WT), and a nominal decrease in ABCG1 and SR-BI expression ( $88 \%$ and $81 \%$, respectively of WT). These values were calculated after correction for the intensities of $\beta$-actin bands, which were identical in both WT and LPL-KD samples. Similar results were obtained in repeated experiments. The effect of LPL silencing on ABCA1 protein expression was investigated by Western blot analysis, but there was no significant difference in the intensity of ABCA1 bands in WT and LPD-KD samples (Figure 2B).

\section{Inverse Correlation between LPL Expression and Cholesterol Efflux}

Of the three cholesterol transporters examined, $\mathrm{ABCA} 1$ and $\mathrm{ABCG} 1$ are unidirectional transporters with $\mathrm{ABCA} 1$ requiring a lipid-poor apoAI as the cholesterol acceptor, and ABCG1 requiring HDL particles. Thus, the ABCA1 pathway results in lipidation of apoAI to form nascent HDL particles, which can then accept cholesterol via the ABCG1 pathway. Since LPL silencing resulted in the greatest modulation of ABCA1 expression, the correlation between LPL expression and ABCA1-mediated cholesterol efflux was investigated. In the absence of extracellular cholesterol acceptors, passive aqueous diffusion of cholesterol was similar in WT and LPL-KD THP-1 cells. In the presence of apoAI, the acceptor for ABCA1-mediated cholesterol efflux, LPL-KD cells demonstrated significantly greater efflux than that in WT cells (Figure 3A). ABCA1-mediated cholesterol efflux (Figure 3B) was calculated as the difference in efflux values in the presence and absence of apoAI and was 4.5-fold greater in LPL-KD THP-1 cells compared to WT cells. These data 
clearly demonstrated an inverse relationship between LPL expression and ABCA1-mediated cholesterol efflux.

Next, to confirm a role for LPL in down-regulating cholesterol efflux, LPL was added back to the culture media of LPL-KD cells. This should mimic the cellular expression of LPL, since LPL is a secreted protein. As expected, addition of exogenous LPL resulted in LPLKD cells reverting to a lower level of cholesterol efflux. In the presence of exogenous LPL, cholesterol efflux to apoAI acceptors was decreased in both WT and LP-KD cells (Figure 4A). Interestingly, aqueous diffusion of cholesterol, measured in the absence of apoAI, was also lower in LPL-KD cells and it was further down-regulated when exogenous LPL was added. The difference in efflux in the presence and absence of apoAI was calculated as the ABCA1-mediated efflux, and plotted against the concentration of exogenous LPL added Figure 4B). There was a clear LPL-dose-dependent decrease in ABCA1-mediated cholesterol efflux in LPL-KD cells. Addition of LPL slightly down-regulated efflux in WT cells as well, thus confirming that macrophage LPL levels modulate ABCA1-mediated cholesterol efflux.

\section{DISCUSSION}

This study investigates the role of macrophage lipoprotein lipase in ABCA1-mediated cholesterol efflux, the first and crucial step of reverse cholesterol transport. Two independent approaches were used to modulate macrophage LPL levels. To obtain LPLdeficient cells, short hairpin RNA specific to the LPL gene were transduced into THP-1 macrophages to silence the LPL gene. Almost complete knock-down of LPL expression was established as demonstrated by RT-PCR and Western blot analysis. LPL gene overexpression was also attempted, but since wild-type macrophages already express abundant LPL, this strategy failed to result in a desirable difference in LPL levels in WT and LPL-overexpressing cells. To circumvent this problem, purified bovine LPL [14] protein was added to the culture media of WT and LPL-KD THP-1 cells. Since LPL is a secreted protein, such LPL-add-back experiments served as an appropriate method for measuring LPL-dose-dependent modulation of cholesterol efflux.

Our results clearly demonstrate that $\mathrm{ABCA} 1$ expression and function is inversely regulated by macrophage LPL levels. These data are consistent with previous evidence that macrophage LPL is proatherogenic. Two studies reported that macrophage-specific depletion of LPL in mice caused a 52-55\% reduction in aortic lesion size compared with controls [7]. We demonstrated that in male apoE knock-out mice, macrophage-specific overexpression of human LPL resulted in a $51 \%$ increase in aortic lesion size after 3 weeks on a high-fat diet [12]. LPL's atherosclerosis-promoting functions are believed to be due to its ability to serve as an adapter between remnant particles and lipoprotein receptors, thereby contributing to receptor-mediated cholesterol uptake [15]. LPL's lipolytic activity may further facilitate cholesterol uptake by unmasking apoE of lipoprotein particles in vivo and increasing their receptor-binding affinity [14]. Since cellular cholesterol-loading is known to dramatically induce ABCA1 transcription due to activation of the LXRa pathway [16], LPL's ability to promote lipoprotein uptake is unlikely to explain our results. Instead, one 
would expect LPL silencing to down-regulate ABCA1 due to the absence of cholesterol loading.

There is likely another mechanism that contributes to the observation that LPL silencing leads to ABCA1 up-regulation. Several studies have implicated fatty acids in transcriptional and post-transcriptional regulation of macrophage ABCA1 [17-19]. Unsaturated fatty acids were shown to negatively modulate ABCA 1 mRNA and protein levels, and diminish ABCA1-mediated cholesterol efflux in RAW264.7 [18] and human monocyte-derived macrophages [19]. This effect also appears to be mediated by the liver X receptor (LXR) since it was abolished in the presence of a LXR agonist, or when the LXRE on the ABCA1 gene was altered by mutagenesis [20]. In addition to decreasing transcription of ABCA1, unsaturated fatty acids also increase ABCA1 protein degradation [17]. It was shown that serine phosphorylation of a PEST sequence in the ABCA1 protein is responsible for its destabilization and rapid turnover [21]. Based on this evidence, it appears that, in our study, silencing the LPL gene results in a depletion of unsaturated fatty acids and removes the down-regulation of ABCA1. When LPL is added back, the unsaturated fatty acid products of lipolysis are replenished, resulting in a reduction in ABCA1-mediated cholesterol efflux.

Western blot analysis failed to show any up-regulation of ABCA1 protein, in spite of an increase in ABCA1 mRNA and ABCA1 function (increased cholesterol efflux). Previous studies have reported similar inconsistency between ABCA1 protein levels and its activity/ function. In a recent publication, Mogilenko et al. showed that ABCA1 protein levels were inversely related to ABCA1 efflux activity [22]. ABCA1 protein has a half-life of approximately one to two hours and its degradation is highly regulated, particularly by LXR $\beta$ [23]. In unstimulated cells, when LXR $\beta$ binds to ABCA1, it slows down ABCA1 degradation, thereby increasing the level of detectable ABCA1 protein. However, this LXR $\beta$-bound ABCA1 cannot participate in cholesterol efflux. When the LXR $\beta / R X R$ complex is activated by its ligand, it results in dissociation of the ABCA1 protein from the nuclear receptor allowing ABCA1 to function in cholesterol efflux but also resulting in its rapid degradation [23]. It is hypothesized that the inverse correlation between ABCA1 function and its degradation may be due to the internalization of apoAI-bound ABCA1 [22]. Such a mechanism may explain our data that LPL-KD macrophages exhibit increased cholesterol efflux, without an increase in ABCA1 protein.

Previously, other members of the lipase gene family were shown to regulate ABCA1 expression and function [24-26]. Qiu and Hill used Lentivirus to over-express or suppress endothelial lipase (EL) in THP-1 macrophages and demonstrated a direct correlation between EL expression levels and ABCA1- dependent cholesterol efflux [25]. EL is primarily a phospholipase and has very little triglyceride lipase activity [26]. The phospholipase activity of EL is responsible for the hydrolysis of HDL phospholipids, thereby lowering HDL levels. In contrast, LPL activity is associated with biogenesis of HDL particles [7]. Thus, the opposite phenotypes obtained by overexpression of EL and LPL are consistent with their primary enzymatic roles.

In a separate study, over-expression of hormone-sensitive lipase (HSL) in THP-1 macrophages increased ABCA1 mRNA as well as cholesterol efflux [27]. HSL is a 
triglyceride lipase, but it does not belong to the lipase gene family. HSL is an intracellular enzyme with a role in fuel metabolism rather than lipoprotein restructuring. It also contains cholesterol ester hydrolase activity. Thus, HSL-overexpressing cells showed an induction in ABCA1 gene expression due to the generation of free cholesterol by HSL-mediated hydrolysis of cellular cholesterol esters.

In conclusion, it is clear that lipases are key players in the modulation of reverse cholesterol transport from macrophages [24,26]. They exert their effect on the expression and function of the cholesterol transporter ABCA1, as well as on modifying the extracellular cholesterol acceptors: nascent HDL or lipid-poor apoAI. Our data demonstrate a clear inverse relationship between macrophage LPL and ABCA1 expression and function. This finding further fuels the need to find therapeutic approaches to inhibit/inactivate macrophage LPL for the prevention/management of atherosclerosis.

\section{Acknowledgments}

This work was supported by National Institutes of Health Awards R15HL083946 and SC3GM095413.

\section{Abbreviations}

ABCA1 ATP-binding cassette transporter A1

LPL lipoprotein lipase

LPL-KD LPL knock-down

LXR liver X receptor

PMA phorbol 12-myristate 13-acetate

PPAR peroxisome proliferator-activated receptor

RXR retinoic-X receptor

ShRNA short hairpin RNA

SR-BI scavenger receptor type BI

WT wild-type

\section{REFERENCES}

1. Libby P, Ridker PM, Hansson GK. Progress and challenges in translating the biology of atherosclerosis. Nature. 2011; 473:317-325. [PubMed: 21593864]

2. Yancey PG, Bortnick AE, Kellner-Weibel G, et al. Importance of different pathways of cellular cholesterol efflux. Arterioscler Thromb Vasc Biol. 2003; 23:712-729. [PubMed: 12615688]

3. Oram JF. HDL apolipoproteins and ABCA1: partners in the removal of excess cellular cholesterol. Arterioscler Thromb Vasc Biol. 2003; 23:720-727. [PubMed: 12615680]

4. Lawn RM, Wade DP, Garvin MR, et al. The Tangier disease gene product ABC1 controls the cellular apolipoprotein-mediated lipid removal pathway. J Clin Invest. 1999; 104:R25-R31. [PubMed: 10525055]

5. Oram JF, Lawn RM, Garvin MR, Wade DP. ABCA1 is the cAMP-inducible apolipoprotein receptor that mediates cholesterol secretion from macrophages. J Biol Chem. 2000; 275:34508-34511. [PubMed: 10918070] 
6. Chawla A, Boisvert WA, Lee CH, et al. A PPAR gamma-LXR-ABCA1 pathway in macrophages is involved in cholesterol efflux and atherogenesis. Mol Cell. 2001; 7:161-171. [PubMed: 11172721]

7. Kersten S. Physiological regulation of lipoprotein lipase. Biochim Biiophy Acta. 2014; 1841:919933.

8. O'Brien KD, Gordon D, Deeb S, et al. Lipoprotein lipase is synthesized by macrophage-derived foam cells in human coronary atherosclerotic plaques. J. Clin. Invest. 1992; 89:1544-1550. [PubMed: 1569193]

9. Babaev VR, Patel MB, Semenovich CF, et al. Macrophage lipoprotein lipase promotes foam cell formation and atherosclerosis in low density lipoprotein receptor-deficient mice. J. Biol. Chem. 2000; 275:26293-26299. [PubMed: 10858435]

10. Ichikawa T, Liang J, Kitajima S, et al. Macrophage-derived lipoprotein lipase increases aortic atherosclerosis in cholesterol-fad rabbits. Atherosclerosis. 2005; 179:87-95. [PubMed: 15721013]

11. Van Eck M, Zimmermann R, Groot PH, et al. Role of macrophage-derived lipoprotein lipase in lipoprotein metabolism and atherosclerosis. Arterioscler. Thromb. Vasc. Biol. 2000; 20:e53-e62. [PubMed: 10978269]

12. Wilson K, Fry GL, Chappell DA, Sigmund CD, Medh JD. Macrophage-specific expression of human lipoprotein lipase accelerates atherosclerosis in transgenic apolipoprotein E knockout mice but not in C57BL/6 mice. Arterioscler Thromb Vasc Biol. 2001; 21:1809-1815. [PubMed: 11701470]

13. Livak KJ, Schmittgen TD. Analysis of relative gene expression data using real-time quantitative PCR and the $2^{-\Delta \Delta C}$ T method. Methods. 2001; 25:402-408. [PubMed: 11846609]

14. Medh JD, Fry GL, Bowen SL, Ruben S, Wong H, Chappell DA. Lipoprotein lipase-and hepatic triglyceride lipase- promoted very low density lipoprotein degradation proceeds via an apolipoprotein E-dependent mechanism. J Lipid Res. 2000; 41:1858-1871. [PubMed: 11060356]

15. Ostlund-Lindqvist AM, Gustafson S, Lindqvist P, Witztum JL, Little JA. Uptake and degradation of human chylomicrons by macrophages in culture. Role of lipoprotein lipase. Arteriosclerosis. 1983; 3:433-440. [PubMed: 6626033]

16. Costet P, Luo Y, Wang N, Tall AR. Sterol-dependent transactivation of the ABC1 promoter by the liver X receptor/retinoid X receptor. J Biol Chem. 2000; 275:28240-28245. [PubMed: 10858438]

17. Lee J, Park Y, Koo SI. ATP-binding cassette transporter A1 and HDL metabolism: effects of fatty acids. J. Nutri. Biochem. 2012; 23:1-7.

18. Ku CS, Park Y-K, Coleman SL, Seo JM, Lee J-Y. Regulation of ATP binding cassette transporter A1 (ABCA1) and ABCG1 by fatty acids in RAW 264.7 macrophages. FASEB J. 2010; 24:924.3.

19. Uehara Y, Engel T, Li Z, et al. Polyunsaturated fatty acids and acetoacetate downregulate the expression of the ATP-binding cassette transporter A1. Diabetes. 2002; 51:2922-2928. [PubMed: 12351428]

20. Uehara Y, Miura SI, von Eckardstein A, et al. Unsaturated fatty acids suppress the expression of the ATP-binding cassette transporter G1 (ABCG1) and ABCA1 genes via an LXR/RXR responsive element. Atherosclerosis. 2007; 191:11-21. [PubMed: 16730733]

21. Wang Y, Oram JF. Unsaturated fatty acids inhibit cholesterol efflux from macrophages by increasing degradation of ATP-binding cassette transporter A1. J Biol Chem. 2002; 277:56925697. [PubMed: 11741998]

22. Mogilenko DA, Shavva VS, Dizhe EB, Orlov SV. PPAR- $\gamma$ activates ABCA1 gene transcription but reduces the level of ABCA1 protein in HepG2 cells. Biochem Biophys Res Commun. 2010; 402:477-482. [PubMed: 20951680]

23. Hozoji M, Munehira Y, Ikeda Y, et al. Direct interaction of nuclear liver X receptor-beta with ABCA1 modulates cholesterol efflux. J. Biol Chem. 2008; 283:30057-30063. [PubMed: 18782758]

24. Annema W, Tietge UJF. Role of hepatic lipase and endothelial lipase in high-density lipoproteinmediated reverse cholesterol transport. Curr Atheroscler Rep. 2011; 13:257-265. [PubMed: 21424685]

25. Qui G, Hill JS. Endothelial liapse promotes apolipoprotein AI-mediated cholesterol efflux in THP-1 macrophages. Arterioscl. Thromb. Vascul Biol. 2009; 29:84-91. 
26. Jin W, Marchadier D, Rader DJ. Lipases and HDL metabolism. Trends Endocrinol. Metab. 2002; 13:174-178. [PubMed: 11943562]

27. Tazoe F, Yagyu H, Okazaki H, et al. Induction of ABCA1 by overexpression of hormone-sensitive lipase in macrophages. Biochem Biophys Res Comm. 2008; 376:111-115. [PubMed: 18762171] 


\section{Highlights}

- The lipoprotein lipase (LPL) gene was successfully silenced in THP-1 macrophages using siRNA.

- ABCA1 transcript levels were increased in LPL-knock-down cells compared to wild-type cells.

- LPL gene silencing resulted in an increase in ABCA1-mediated cholesterol efflux (ACE).

- Replenishing LPL in LPL-KD cells resulted in a LPL-dose-dependent decrease in ACE.

- Our data demonstrate an inverse correlation between macrophage LPL levels and ACE. 
A.
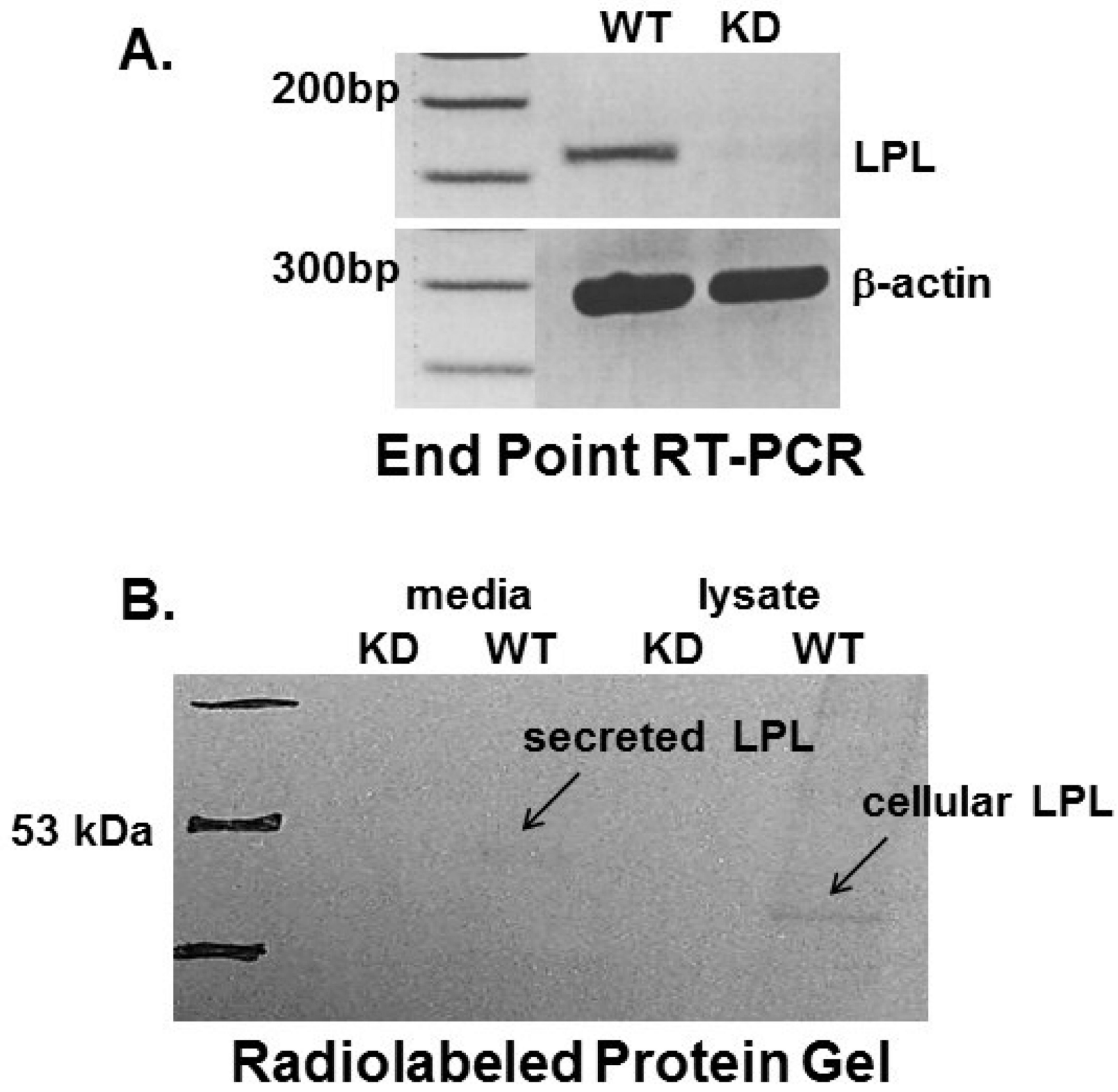

Figure 1. Knock-down of $L P L$ gene expression in THP-1 cells using shRNA Lentivirus particles Lentiviral Particles (Santacruz Biotechnology) were used to deliver a plasmid encoding LPL-specific shRNA to THP-1 monocytes. Cells with stable expression of shRNA were selected by puromycin treatment. A. End-point RT-PCR analysis shows successful knockdown of LPL mRNA levels by $89 \%$ in shRNA transduced monocytes (KD) compared to wild-type (WT) cells. $\beta$-actin was amplified as a control for shRNA specificity, RNA quantification, and gel loading. B. SDS-PAGE and autoradiography of radiolabelled de novo protein synthesis show a decrease in (or near absence of) cellular (lysates) and secreted 
(media) LPL protein in LPL-KD THP-1 cells compared to WT cells. The left-most lane shows the positions of pre-stained low-range molecular weight markers (BioRad). 


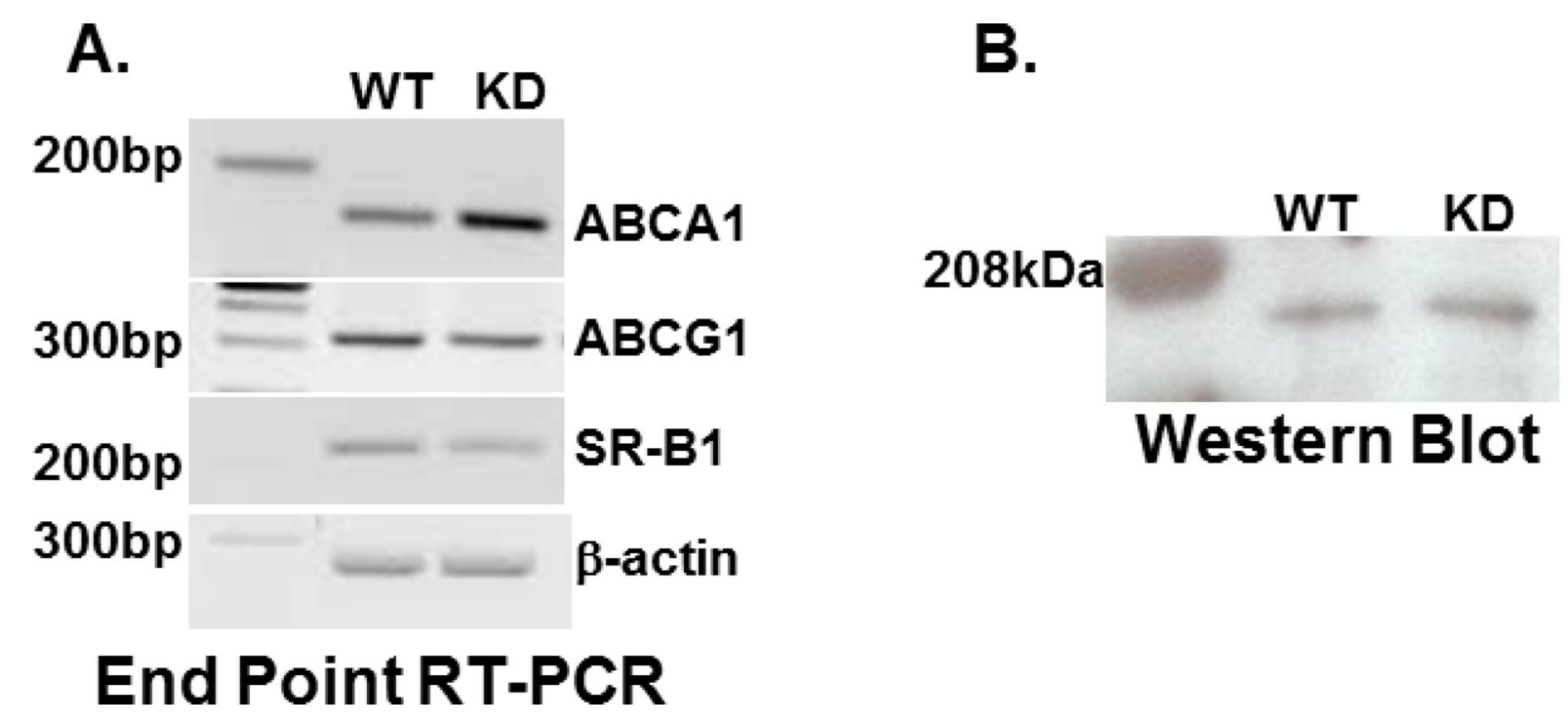

Figure 2. Down-regulation of LPL expression induces ABCA1 transcription without a detectable increase in ABCA1 protein

A. Total RNA isolated from differentiated LPL-KD and WT THP-1 macrophages was subjected to end-point RT-PCR analysis using primers specific for cholesterol transporters ABCA1, ABCG1, SR-B1 (Table 1). $\beta$-actin was amplified as a control for RNA quantification and gel loading. B. Cell lysates were prepared from differentiated WT and LPL-KD THP-1 macrophages and total protein was quantified by the Lowry assay. Equal amounts of protein were resolved by $8 \%$ SDS-PAGE and ABCA1 protein was detected by Western blot analysis using a mouse anti-ABCA1 primary antibody (Novus Biologicals) and a HRP-conjugated goat anti-mouse secondary antibody. A chemiluminescent substrate (SuperSignal West Pico from Thermo Scientific) followed by autoradiography enabled visualization of ABCA1 protein. 

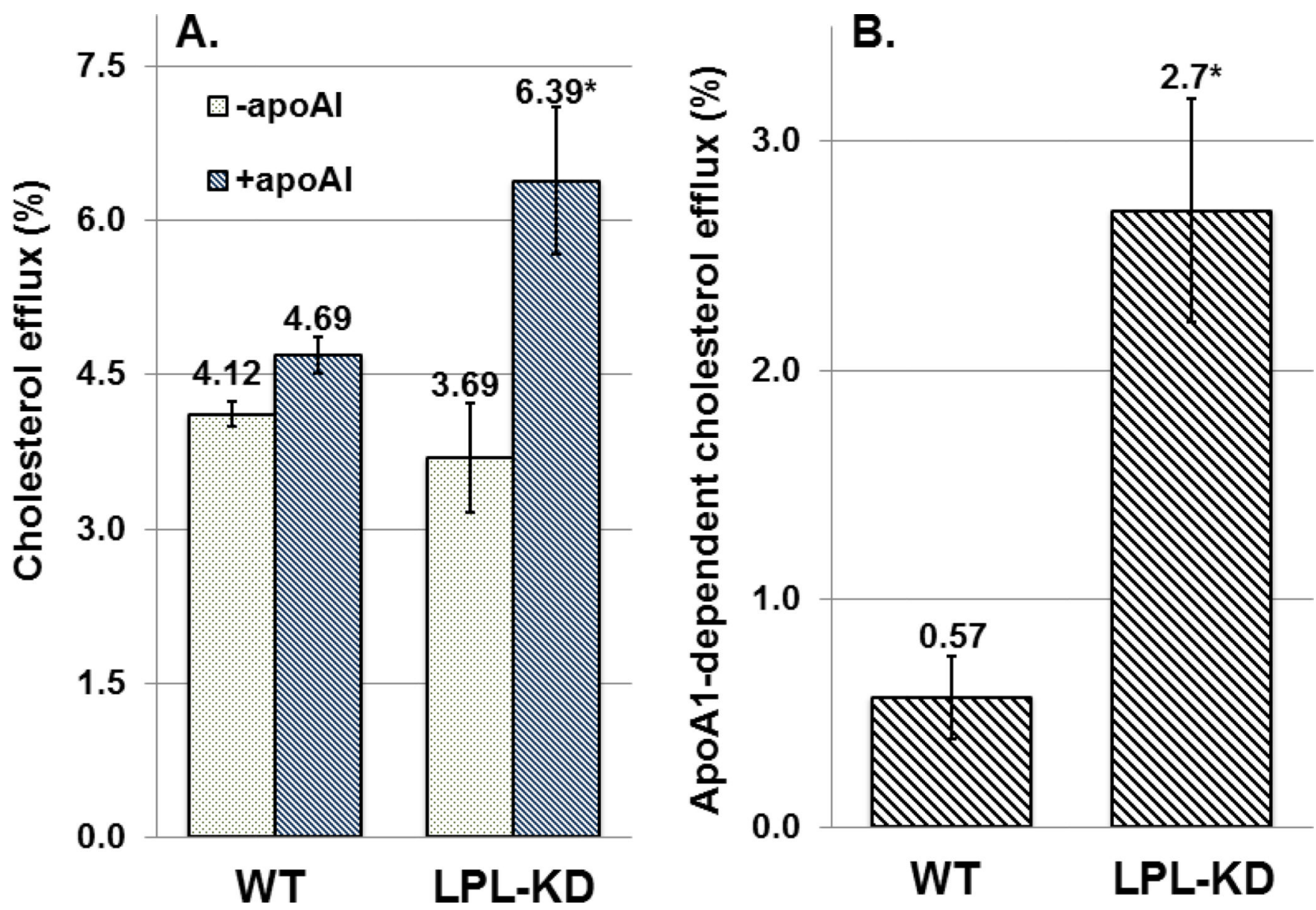

Figure 3. Suppression of macrophage-derived LPL correlates with an increase in ABCA1mediated cholesterol efflux

The efflux of $\left[{ }^{3} \mathrm{H}\right]$-cholesterol from differentiated WT and LPL-KD THP-1 macrophages was measured in the absence or presence of $32.7 \mu \mathrm{g} / \mathrm{mL}$ ApoA-I for 12 hours. Radioactivity in the culture media and cellular lipid extracts was determined by scintillation counting and cholesterol efflux was calculated as $(\%$ efflux $=[\mathrm{cpm}$ in media $\times 100] /[\mathrm{cpm}$ in media $+\mathrm{cpm}$ in lipid extracts]). Each value is an average of triplicates \pm standard deviation. A. Total cholesterol efflux is shown for each dataset. ( ${ }^{*} \mathrm{P}<0.05$ for LPL-KD compared to WT cells). B. ABCA1-dependent cholesterol efflux is calculated as the difference in efflux by aqueous diffusion (absence of ApoA-I) and efflux in the presence of ApoA-I. ( $\left.{ }^{*} \mathrm{P}<0.01\right)$. 

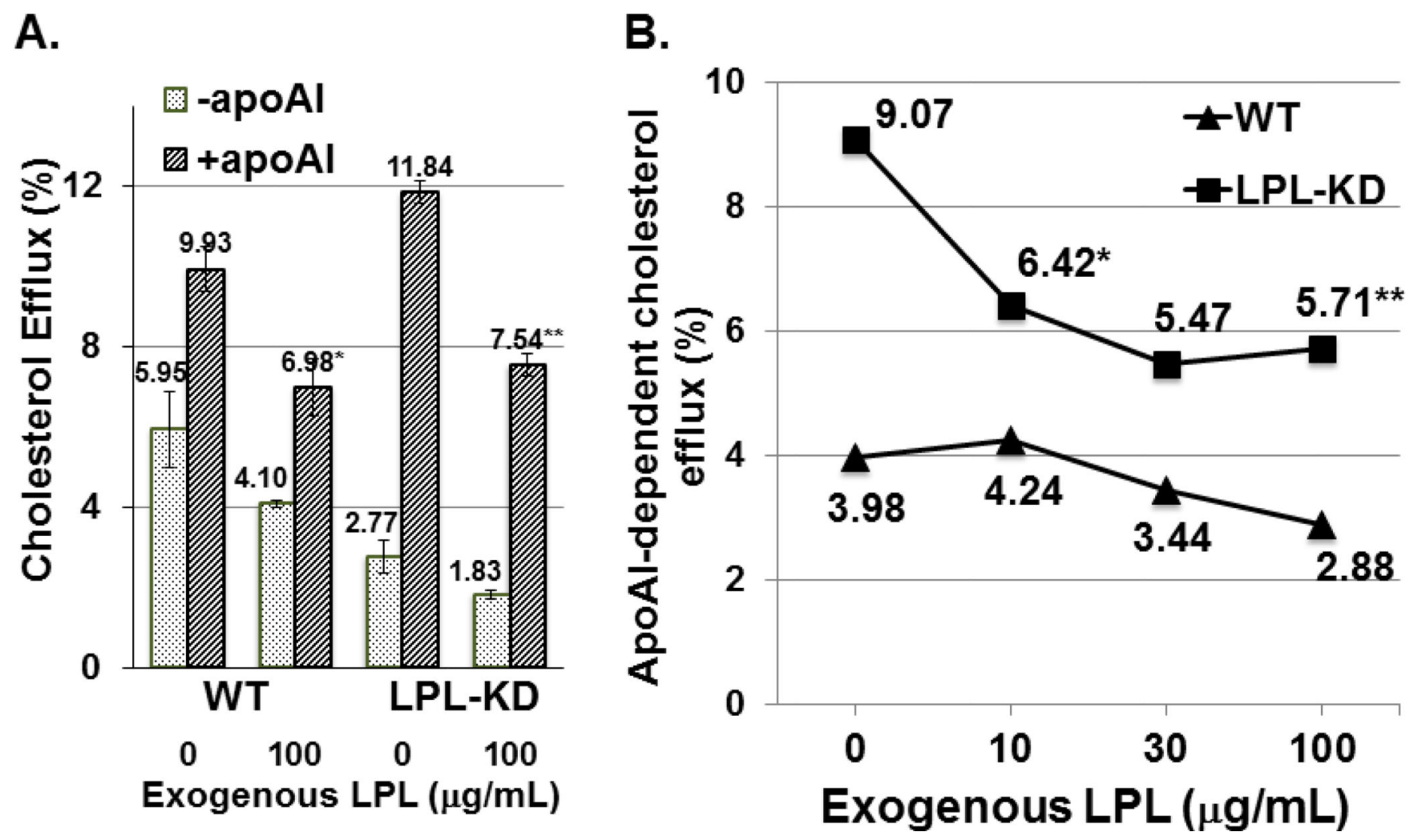

Figure 4. Addition of LPL to THP-1 cell culture medium inhibits apoAI-mediated cholesterol efflux

Cholesterol efflux was measured in LPL-KD and WT THP-1 macrophages as described in Figure 3. During the efflux assay, the specified concentration of purified bovine LPL was added. A. Addition of $100 \mu \mathrm{g} / \mathrm{mL}$ LPL reduces cholesterol efflux in both WT and LPL-KD cells. ( ${ }^{*} \mathrm{P}<0.01$; $^{* *} \mathrm{P}<0.005$ compared to corresponding ' $0 \mu \mathrm{g} / \mathrm{mL}$ LPL'). B. ABCA1dependent cholesterol efflux was calculated as a difference in efflux between the absence and presence of ApoA-I. The $\mathrm{x}$-axis is not to scale. The data points are mean values of triplicate determinations. ( ${ }^{*} \mathrm{P}<0.05$ and ${ }^{* *} \mathrm{P}<0.0005$ compared to corresponding ${ }^{\prime} 0 \mu \mathrm{g} / \mathrm{mL}$ LPL'). 
Table 1

Primer sequences for end-point and real-time PCR

\begin{tabular}{|c|c|c|}
\hline $\begin{array}{c}\text { Gene \& Amplicon } \\
\text { Size (bp) }\end{array}$ & Primer Type & Primer Sequence \\
\hline \multirow{2}{*}{ huLPL 308} & Sense 2 & $5^{\prime}$-GGAATGTATGAGAGTTGGGT-3' \\
\cline { 2 - 3 } & Antisense 2 & $5^{\prime}$-GGGCTTCTGCATACTCAAAG-3' \\
\hline \multirow{2}{*}{ huABCA1 157 } & Sense & $5^{\prime}$-AACAGTTTGTGGCCCTTTTG-3' \\
\cline { 2 - 3 } & Antisense & $5^{\prime}$-AGTTCCAGGCTGGGGTACTT-3' \\
\hline \multirow{2}{*}{ huABCG1 317 } & Sense & $5^{\prime}$-GGTTCTTCGTCAGCTTCGAC-3' \\
\cline { 2 - 3 } & Antisense & $5^{\prime}$-GTTTCCTGGCATTCAGGTGT-3' \\
\hline \multirow{2}{*}{ huSR-BI 216 } & Sense & $5^{\prime}$-CTGTGGGTGAGATCATGTGG-3' \\
\cline { 2 - 3 } & Antisense & 5'-GCCAGAAGTCAACCTTGCTC-3' \\
\hline \multirow{2}{*}{-actin 285 } & Sense & $5^{\prime}$-TCATGAAGTGTGACGTTGACATCCGT-3' \\
\cline { 2 - 3 } & Antisense & 5'-CTTAGAAGCATTTGCGGTGCACGATG-3' $^{\prime}$ \\
\hline
\end{tabular}




\section{Table 2}

Real-time PCR $\mathrm{C}_{\mathrm{T}}$ values

\begin{tabular}{|c|c|c|}
\hline & Wild-Type & LPL-KD \\
\hline$\beta$-actin & 16.55 & 16.17 \\
\hline LPL & 20.11 & 28.11 \\
\hline Fold change & & $\mathbf{0 . 0 0 3}$ \\
\hline
\end{tabular}

